\title{
SOST Gene Inhibits Osteogenesis from Adipose-Derived Mesenchymal Stem Cells by Inducing Th17 Cell Differentiation
}

\author{
Li You Lin Chen Ling Pan Yongde Peng Jinyu Chen \\ Department of Endocrinology and Metabolism, Shanghai First People's Hospital, School of Medicine, \\ Shanghai Jiao Tong University, Shanghai, China
}

\section{Key Words}

Sclerostin • ADSCs • Th17 cells $・$ IL-17 • Osteogenesis

\begin{abstract}
Background/Aims: Postmenopausal osteoporosis is considered to be an autoimmune and inflammatory process, and IL-17 plays important roles in the loss of bone mass. Sclerostin (SOST) acts as a negative regulator of bone formation by inhibiting the Wnt signaling pathway. It also is a mediator of the crosstalk between the skeletal and immune systems. However, few studies have examined the role of SOST gene in the differentiation of T helper 17 (Th17) cells. Methods: Adipose-derived stem cells (ADSCs) were isolated and transfected with pcDNA3SOST or shSOST, and then co-cultured with $\mathrm{CD}^{+}{ }^{+} \mathrm{T}$ cells isolated from peripheral blood mononuclear cells. The differentiation, adipogenesis, and osteogenesis of Th17 and regulatory $\mathrm{T}$ (Treg) cells were examined by western blot, intracellular and intranuclear staining, ELISA, and real-time quantitative PCR in this co-culture model. Results: The SOST gene promoted the secretion of IL- 6 and TGF- $\beta$ in ADSCs. After co-culture of ADSCs with CD4 ${ }^{+}$T cells, the SOST gene increased the number of CD4 ${ }^{+} \mathrm{IL}-17^{+}$cells and the levels of IL-17 and ROR $\gamma$. However, the number of $\mathrm{CD} 4^{+} \mathrm{CD} 25^{+} \mathrm{Foxp}^{+}$cells was decreased, which was accompanied with a reduction of IL-10 and Foxp3 expression. In the meantime, the SOST gene inhibited the expression of COL1, OCN, and OPN, reduced the activity of alkaline phosphatase, and increased the expression of LPL and PPARY. Furthermore, IL-17 promoted SOST gene-induced adipogenesis and increased the inhibition of osteogenesis. Conclusions: SOST promoted the differentiation of Th17 cells and reduced the differentiation of Treg cells, which exacerbated the SOST geneinduced inhibition of osteogenesis from ADSCs.
\end{abstract}

(C) 2018 The Author(s)

Published by S. Karger AG, Basel

\section{Introduction}

Osteoporosis is defined as a systemic skeletal disease caused by an imbalance between bone formation and resorption, and is characterized by low bone mass and microarchitectural deterioration of bone tissue [1]. Adipose-derived stem cells (ADSCs) are an attractive source

\begin{tabular}{ll}
\hline Li You & Dept. of Endocrinology and Metabolism, Shanghai First People's Hosp., School of Medicine \\
& Shanghai Jiao Tong University, Shanghai, 200080 (China) \\
& E-Mail youlisky2002@126.com
\end{tabular}


of cells for the regeneration of damaged tissues because of their capacity for self-renewal and differentiation into various cell lineages, such as adipogenic, osteogenic, myogenic, and chondrogenic lineages, and their use in the treatment of skeletal defects including osteoporosis has been investigated extensively [2, 3]. Specifically, mesenchymal stem cells (MSCs) have been shown to possess anti-inflammatory and immunomodulatory properties mainly due to their inhibitory effect on the proliferation of T cells [4].

The close relationship between the skeletal and immune systems has been increasingly recognized [5]. Postmenopausal osteoporosis is now hypothetically considered to be an autoimmune and inflammatory process in which many pro-inflammatory and $\mathrm{T}$ cell-derived cytokines play important roles in the loss of bone mass [5]. Inflammation increases the production of tumor necrosis factor (TNF) or receptor activator of NF-kB ligand (RANKL) by activated T cells, which is linked to bone loss-associated diseases [6]. The Th17 cell effector phenotype is defined by the preferential secretion of interleukin (IL)-17A, along with other cytokines. T helper 17 (Th17) cells are pro-inflammatory and protect against extracellular pathogens [4]. IL-17 has been shown to be an important mediator of inflammatory arthritis and other diseases affecting bone. Th17 T cells have been suggested to be osteoclastogenic $\mathrm{T}$ cells that are induced by high levels of RANKL and IL-17 [7]. Serum IL-17A concentration was shown to be significantly higher in a postmenopausal osteoporosis group than in a normal postmenopausal group, and treatment with bisphosphonates reduced Th17 cell cytokine levels $[8,9]$. These results indicated that activated Th17 cells induce the production of IL17 and lead to bone resorption. MSCs exert broad immunomodulatory effects, and they are also an important resource for osteogenesis. Interestingly, Ghannam et al. showed that MSCs inhibited the differentiation and function of Th17 cells, and induced the differentiation of the regulatory $\mathrm{T}$ (Treg) cell phenotype [10].

Sclerostin (SOST) acts as a negative regulator of bone formation through inhibition of the Wnt signaling pathway, which is of critical importance for the development and function of osteoblasts [11]. The downregulation of SOST gene is associated with increased osteogenesis and bone mass [12]. SOST inhibits the action of bone morphogenetic protein (BMP) signaling by binding to Wnt co-receptors or low-density lipoprotein receptors (LRP) 5 and 6 [13]. Compounds that inhibit SOST have been shown to stimulate bone formation and reduce bone resorption, with a robust increase in bone mineral density [14]. In our previous study, we found that SOST inhibited the osteogenic activity of ADSCs. Zfp467 binds to and regulates the expression of the SOST gene and promotes the binding of Sost to the Wht co-receptors LRP5/6 [15]. SOST is a mediator of the crosstalk between the skeletal and immune systems [16]. However, there is little information on the relationship between SOST and Th17 cells in bone formation.

On the basis of the roles of SOST gene in the osteogenic activity of MSCs and regulation of immune activity, we investigated the effect of SOST gene on the differentiation and function of Th17 cells by co-culturing ADSCs with CD4 ${ }^{+} \mathrm{T}$ cells. In this study, we showed that SOST gene promoted the production of IL- 6 and transforming growth factor (TGF)- $\beta$ in ADSCs, which induced the differentiation and function of Th17 cells and reduced the differentiation of Treg cells.

\section{Materials and Methods}

Isolation and culture of ADSCS

Abdominal adipose tissues were obtained from 6-8-week-old female BALB/C mice following the Animal Research Guidelines of Shanghai Jiao Tong University School of Medicine. The investigation conformed to the "Guide for the Care and Use of Laboratory Animals" published by the National Institutes of Health of the United States of America and was approved by the Ethics Committee for the Use of Human or Animal Subjects of Shanghai Jiao Tong University. The lipoaspirate was incubated with collagenase type I solution (Worthington Biochemical, Lakewood, NJ) for $1 \mathrm{~h}$ at $37^{\circ} \mathrm{C}$, filtered through $250-\mu \mathrm{m}$ filters, and centrifuged. The stromal vascular fraction was resuspended in Dulbecco's modified Eagle's medium (HyClone, Logan, UT) 
supplemented with $10 \%$ fetal bovine serum (HyClone), $100 \mathrm{U} / \mathrm{mL}$ penicillin, and $100 \mu \mathrm{g} / \mathrm{mL}$ streptomycin. ADSCs were cultured under a humidified atmosphere of $5 \% \mathrm{CO}_{2}$ at $37^{\circ} \mathrm{C}$ and showed the capacity to differentiate into adipocytes or osteogenic cells in the presence of induction factors as described previously [17]. For bone formation, the cells were induced with $0.1 \mu \mathrm{mol} / \mathrm{L}$ hexadecadrol, $50 \mu \mathrm{mol} / \mathrm{L}$ vitamin C, and $10 \mathrm{mmol} / \mathrm{L}$ phosphoglycerol. For adipogenesis, the cells were induced with $1 \mu \mathrm{mol} / \mathrm{L}$ hexadecadrol, 0.5 mmol/L IBMX, and $200 \mu \mathrm{mol} / \mathrm{L}$ indomethacin. For identification, ADSCs were stained with anti-CD29 and anti-CD44 antibodies (eBioscience, San Diego, CA) and tested by flow cytometry. Differentiation of ADSCs into adipocytes and osteoblasts was monitored by oil red $O$ staining of lipid droplets and von Kossa staining of calcium deposits in the extracellular matrix, respectively.

\section{Vector construction and transient transfection assay}

The Sost gene was generated by PCR and cloned into the EcoR1 and BamH1 sites of the pCDNA3.1 vector (Promega, Madison, WI). Primer sequences were designed based on the published sequence of the Sost gene. ADSCs were cultured as described above, plated in 12-well plates, and grown to 60-80\% confluence before transfection using Lipofectamine ${ }^{\circledR} 2000$ (Invitrogen, Carlsbad, CA) according to the manufacturer's instructions.

\section{Small interfering RNA vector construction}

The SOST-short hairpin RNA (shRNA) vector was generated using the pGMLV-SC5 plasmid and verified by sequencing. For silencing of Sost, ADSCs were transfected with shRNA against the mouse Sost gene using Lipofectamine 2000 (Invitrogen). The expression of the SOST gene was analyzed by western blotting and real-time quantitative PCR (RT-qPCR).

\section{Th17 and Treg differentiation and ADSC co-cultures}

Female BALB/C mice (6-8 weeks old) were anesthetized and sacrificed. Peripheral blood was collected from the angular vein, using $4 \%$ sodium citrate as an anticoagulant. Peripheral blood mononuclear cells (PBMCs) were obtained by density gradient centrifugation over HISTOPAQUE-1083 (Sigma-Aldrich, St. Louis, MO), washed 3 times with RPMI-1640, and counted. CD4+ $\mathrm{T}$ cells were isolated using a CD4+ $\mathrm{T}$ Cell Isolation Kit (Miltenyi Biotec, Bergisch Gladbach, Germany). CD4+ $\mathrm{T}$ cells were stimulated with $2.5 \mu \mathrm{g} / \mathrm{mL}$ anti-CD3 and $1.5 \mu \mathrm{g} / \mathrm{mL}$ anti-CD28 antibodies (Abcam, Cambridge, UK), and subsequent cultured with 20 ng/mL IL-6 and 3 ng/mL TGF- $\beta$ (PeproTech, Rocky Hill, NJ). CD4+ T cells $\left(2.0 \times 10^{5}\right.$ cells) were co-cultured with $2.0 \times 10^{6}$ ADSCs in the presence or absence of SOST or shSOST at days 1,2 , and 4 after CD $4^{+} \mathrm{T}$ cell stimulation.

\section{Intracellular and intranuclear staining}

Intracellular staining of IL-17 in CD4+ $\mathrm{T}$ cells was performed after $6 \mathrm{~h}$ of activation with $1 \mathrm{ng} / \mathrm{mL}$ PMA and $1 \mathrm{mg} / \mathrm{mL}$ ionomycin (Calbiochem, San Diego, CA) in the presence of brefeldin A (10 mg/mL; SigmaAldrich). The cells were then stained with an FITC-conjugated anti-CD4 monoclonal antibody (mAb) for $30 \mathrm{~min}$ at $4^{\circ} \mathrm{C}$, washed with FACS buffer ( $2 \%$ fetal bovine serum, $0.1 \%$ sodium azide in phosphate-buffered saline), treated with Cytofix/Cytoperm for $20 \mathrm{~min}$, and washed with Perm/Wash Buffer (BD Biosciences, San Jose, CA). The cells were subsequently stained with a PE-conjugated anti-mouse IL-17 mAb for $30 \mathrm{~min}$ at $4^{\circ} \mathrm{C}$. Then, the cells were fixed at $4{ }^{\circ} \mathrm{C}$ with $4 \%$ paraformaldehyde. The expression of CD25 and transcription factors was assessed using an FITC-conjugated mAb specific for CD25 and a PE-conjugated mAb specific for fork head box p3 (FOXP3), respectively. Sample data were acquired using a FACSCalibur flow cytometer (BD Biosciences).

\section{Quantification of cytokines}

The culture supernatants were collected, and the production of IL-6, TGF- $\beta$, IL-10, and IL-17 was analyzed using enzyme-linked immunosorbent assay (ELISA) kits (eBioscience, San Diego, CA).

\section{Western blotting}

Cell lysates for immunoblotting were prepared in a lysate buffer containing $20 \mathrm{mM}$ Tris-HCl, $\mathrm{pH}$ 7.5, 150 mM NaCl, 1\% NP-40, 0.5\% Na-deoxycholate, 1 mM EDTA, 0.1\% SDS, protease inhibitors (Roche Molecular Biochemicals, Mannheim, Germany), $1 \mathrm{mM} \mathrm{Na}_{3} \mathrm{VO}_{4}$, and $1 \mathrm{mM} \mathrm{NaF}$. Protein (20 $\mu \mathrm{g}$ ) was separated on a 
8-12\% NuPAGE® Novex®Bis-Tris gel system (Invitrogen). The membrane was blocked and probed with anti-SOST or anti-GAPDH antibodies (Abcam) and incubated with a peroxidase-conjugated secondary antibody. Bands were visualized by detection with the ECL system (Amersham, Piscataway, NJ).

\section{$R T-q P C R$}

Total RNA was isolated using the TRIzol reagent (Invitrogen) according to the manufacturer's instructions. RT-qPCR was performed using an ABI StepOne $^{\mathrm{TM}}$ Real-Time PCR machine with Fast SYBR® Green Master Mix (Applied Biosystems, Foster City, CA). The primers used are listed in Table 1.

\section{Alkaline phosphatase activity analysis}

Alkaline phosphatase (ALP) activity was determined following incubation with $1 \mathrm{mg} / \mathrm{mL}$ P-nitrophenyl phosphate in $50 \mathrm{mM} \mathrm{NaHCO}_{3}$ and $1 \mathrm{mM} \mathrm{MgCl}_{2}$ buffer (pH 9.6) at $37^{\circ} \mathrm{C}$ for $20 \mathrm{~min}$. Activity was stopped by the addition of $3 \mathrm{M} \mathrm{NaOH}$. Reaction absorbance was measured at $405 \mathrm{~nm}$ using a FLUO star Omega plate reader, and ALP activity was corrected for cell viability.

\section{Statistical analysis}

All experiments were repeated a minimum of 3 times. Data are reported as the mean \pm standard deviation (SD). Comparisons between groups were made using Student's t test with $\mathrm{p}<0.05$ considered statistically significant.
Table 1. Primers for qPCR

\begin{tabular}{cc}
\hline Gene & Primer (5'-3') \\
\hline IL-17-F & GCCCTCAGACTACCTCAACC \\
IL-17-R & GATGATTTCCCGTGGTGGTC \\
Foxp3-F & GGCAATAGTTCCTTCCCAGA \\
Foxp3-R & TCATTGAGTGTCCTCTGCCT \\
IL-10-F & TGAGAACCAAGACCCAGACA \\
IL-10-R & ACGACCACTGCTTACGGAAA \\
ROR $\gamma-\mathrm{F}$ & CCCGAGATGCTGTCAAGTT \\
ROR $\gamma-\mathrm{R}$ & CACTTGTTCCTGTTGCTGCT \\
OPN-F & TGACGATGATGATGACGATG \\
OPN-R & GGGACGATTGGAGTGAAAGT \\
COL1-F & ACGATGGTGCTAAGGGTGAT \\
COL1-R & GGGACCACTTTCACCTCTGT \\
LPL-F & AAGGGCTCTGCTTGAGTTGT \\
LPL-R & TTGTAGGGCATCCGAGAACG \\
PPAR $\gamma$-F & GCCAGAGTGAGTGCCAGGAC \\
PPAR $\gamma-\mathrm{R}$ & TTCCTCCTCTTCTTCCTCTTCTT \\
OCN-F & CTCACTCTGCTGGCCCTGGC \\
OCN-R & GGGCCGCTGGGCTTGGCATC \\
\hline
\end{tabular}

\section{Results}

Characterization of ADSCs and SOST gene transfection

Mouse ADSCs were isolated and identified by CD29 and CD44 staining. After purification, CD29 and CD44 were expressed in greater than 99\% of mouse ADSCs. The plasmid pcDNASOST and shSOST were transfected into mouse ADSCs, and the protein level of SOST was detected by western blot analysis. As shown in Fig. 1A, pcDNA3-SOST transfection significantly increased the level of SOST protein. However, shSOST transfection remarkably reduced the level of SOST protein (Fig. 1B).

SOST increases the expression and secretion of IL- 6 and TGF- $\beta$ in ADSCS

To observe the effect of SOST on the expression of IL- 6 and TGF- $\beta$, pcDNA-SOST or shSOST was transfected into mouse ADSCs. qPCR analysis indicated that SOST gene overexpression increased the expression of IL-6 and TGF- $\beta$ mRNA (Fig. 2A, 2B), while SOST inhibition significantly reduced their expression. In addition, the secretion of IL- 6 and TGF- $\beta$ was also detected by ELISA, which generated similar results to those of RT-qPCR (Fig. 2C, 2D).

SOST increases the differentiation of Th17 cells with co-culture of ADSCs and CD4+ $T$ cells

On the basis of the above results, we examined the effect of ADSCs on the differentiation of Th17 cells from CD4+ $4^{+}$cells. Mouse ADSCs were transfected with pcDNA3-SOST or shSOST and then co-cultured with CD4+ $\mathrm{T}$ cells. The co-expression of CD4 and IL-17 was tested by intracellular cytokine staining in $\mathrm{CD}^{+}{ }^{+} \mathrm{T}$ cells. As shown in Fig. 3A and 3B, SOST gene overexpression in ADSCs increased the differentiation of CD $4^{+} I L 17^{+} \mathrm{T}$ cells; however, SOST gene knockdown significantly reduced the differentiation of $\mathrm{CD} 4^{+} \mathrm{IL} 17^{+} \mathrm{T}$ cells. To further observe Th17 cell differentiation induced by SOST gene, the cell supernatants were collected and the level of IL-17 was tested by ELISA. Compared with the control group, SOST promoted the secretion of IL-17 from CD4 ${ }^{+} \mathrm{T}$ cells; in contrast, inhibition of SOST gene expression reduced IL-17 secretion (Fig. 3C). In addition, CD4 $4^{+} \mathrm{T}$ cells were collected after co-culture and the expression of IL-17 and retinoid-related orphan receptor gamma (ROR $\gamma$ ) mRNA was 
detected by RT-qPCR. Similar to the above results, SOST increased the expression of IL-17 and ROR $\gamma$ mRNA in CD4 ${ }^{+} \mathrm{T}$ cells, while inhibition of SOST reduced their expression (Fig. 3D, E).

SOST reduces the differentiation of Treg cells with co-culture of ADSCs and CD $4^{+} T$ cells

We further investigated the effect of ADSCs on the differentiation of Treg cells from CD4+ T cells. Mouse ADSCs were transfected with pcDNA3-SOST or shSOST and then co-cultured with $\mathrm{CD} 4^{+} \mathrm{T}$ cells. The co-expression of CD25 and Foxp3 was tested by intracellular cytokine staining in $\mathrm{CD}^{+}{ }^{+} \mathrm{T}$ cells. As shown in Fig. 4A and 4B, SOST over-expression in ADSCs reduced the differentiation of $\mathrm{CD}_{2} 5^{+} \mathrm{Foxp} 3^{+} \mathrm{T}$ cells in $\mathrm{CD} 4^{+} \mathrm{T}$ cells; however, SOST gene knockdown significantly reduced the differentiation of $\mathrm{CD} 25^{+} \mathrm{Foxp} 3^{+} \mathrm{T}$ cells. To further observe Treg cell differentiation induced by SOST the cell supernatants were collected and the level of IL-10 was tested by ELISA. SOST reduced the secretion of IL-10 in CD4 ${ }^{+} \mathrm{T}$ cells compared with the control group; In contrast, inhibition of SOST gene expression increased the secretion of IL-10 (Fig. 4C). In addition, CD ${ }^{+} \mathrm{T}$ cells were collected after co-culture and the expression of IL-10 and Foxp3 mRNA was detected by qPCR. SOST reduced the expression of IL-10 and Foxp3 mRNA in CD4+ $\mathrm{T}$ cells, while inhibition of SOST promoted their expression (Fig. 4D, E).

\section{SOST reduces osteogenesis with co-culture of ADSCs and CD4 ${ }^{+}$T cells}

The results shown above indicated that SOST gene over-expression in ADSCs may promote the differentiation of Th17 cells. Therefore, we also examined the role of Th17 cells in bone formation. After co-culture of ADSCs and CD4 ${ }^{+} \mathrm{T}$ cells, ADSCs were collected and the expression of collagen, osteocalcin (OCN), and osteopontin (OPN) was detected by RT-qPCR. As shown in Fig. 5A, 5B, and 5C, co-culture of SOST-transfected ADSCs and PBMCs reduced the expression of collagen, OCN, and OPN in ADSCs, while co-culture of shSOST-transfected

Fig. 1. Mouse ADSC isolation and SOST expression. Mouse ADSCs were isolated and identified by staining with anti-CD44 and anti-CD29 antibodies (A). CD44 and CD29 expression was detected by flow cytometry. (B) ADSCs were cultured and transfected with pcDNA3-SOST or shSOST, and the expression of SOST was analyzed with western blotting and RT-qPCR analyses. Data are representative of 3 independent experiments and are expressed as the mean \pm SD. $* \mathrm{p}<0.05$.

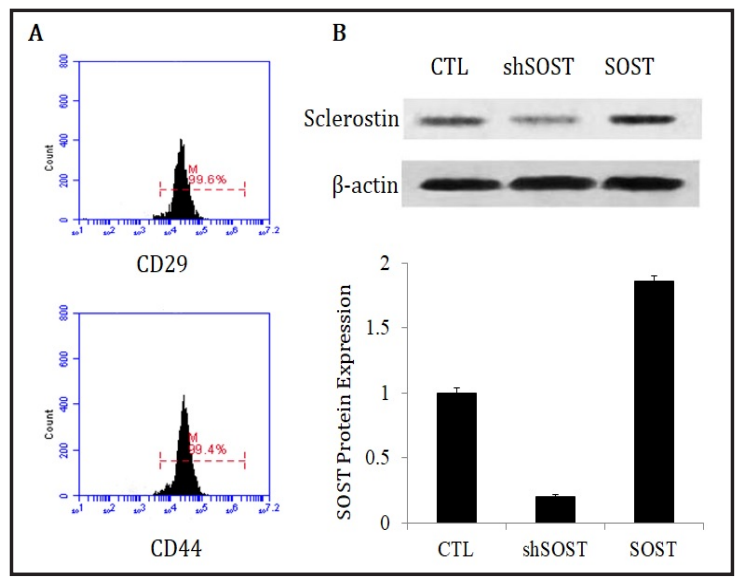

Fig. 2. Effect of SOST on the expression of TGF- $\beta$ and IL-6. Mouse ADSCs were cultured and then transfected with pcDNA3SOST or shSOST. The expression of IL- 6 (A) and TGF- $\beta$ (B) mRNA was analyzed by RT-qPCR. The secretion of IL- 6 (C) and TGF- $\beta$ (D) was detected by ELISA. Data are representative of 3 independent experiments and are expressed as the mean \pm SD. ${ }^{*} \mathrm{p}<0.05$ vs. control group; ${ }^{* *} \mathrm{p}<0.01$ vs. control group.

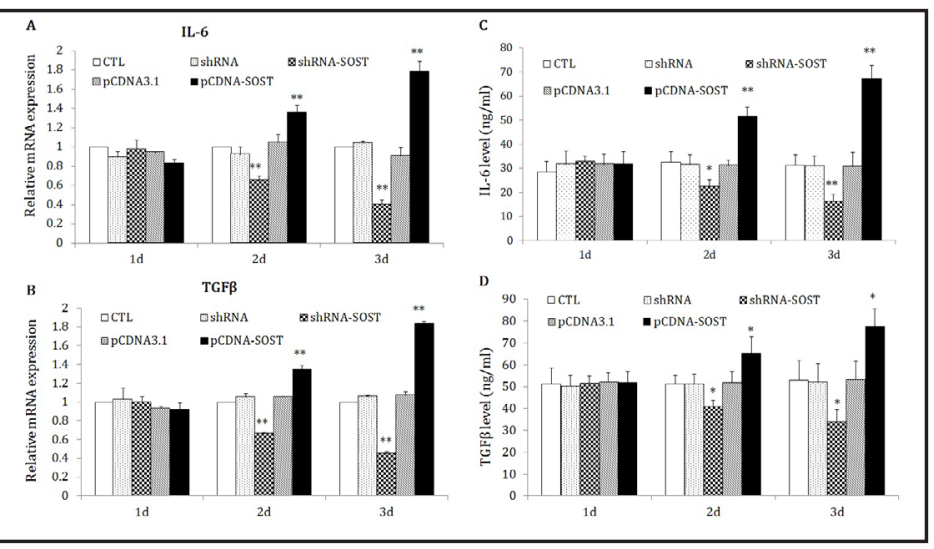


ADSCs and CD4 ${ }^{+} \mathrm{T}$ cells increased their expression. The ALP activity of ADSCs also tested and showed similar trends with the above results (Fig. 5D).

SOST promotes adipogenic differentiation with co-culture of ADSCs and CD4+ T cells

Furthermore, the adipogenic differentiation activity of ADSCs was detected after co-culture with $\mathrm{CD} 4^{+} \mathrm{T}$ cells. The expression of lipoprotein lipase (LPL) and peroxisome proliferator-activated receptor gamma (PPAR $\gamma$ ) was investigated by RT-qPCR. Co-culture of SOST-transfected ADSCs and CD4 ${ }^{+} \mathrm{T}$ cells increased the expression of LPL and PPAR $\gamma$ mRNA in ADSCs, while SOST gene knockdown in ADSCs reduced their expression after co-culture with $\mathrm{CD}^{+}{ }^{+} \mathrm{T}$ cells (Fig. 6A, B).

Fig. 3. Effect of SOST on the differentiation of Th17 cells. Mouse PBMCs were prepared and $\mathrm{CD}^{+} \mathrm{T}$ cells were purified and then stimulated with antibodies and cytokines. CD4 ${ }^{+} \mathrm{T}$ cells were co-cultured with ADSCs and then collected at days 0,2 , and 4 for further analysis. The percentage of $\mathrm{CD}^{+} \mathrm{IL}^{-17^{+}} \mathrm{T}$ cells was tested by intracellular staining (A, B). The level of IL-17 was detected by ELISA (C). The expression of IL-17 (D) and ROR $\gamma$ (E) mRNA was tested by RT-qPCR. Data are representative of 3 independent experiments and are expressed as the mean \pm SD. ${ }^{*} \mathrm{p}<0.05$ vs. control group; ${ }^{* *} \mathrm{p}<0.01$ vs. control group.

Fig. 4. Effect of SOST on the differentiation of Treg cells. Mouse PBMCs were prepared and $\mathrm{CD}^{+}$ $\mathrm{T}$ cells were purified and then stimulated with antibodies and cytokines. $\mathrm{CD}^{+} \mathrm{T}$ cells were cocultured with ADSCs and then collected at days 0,2 , and 4 for further analysis. The percentage of $\mathrm{CD}_{25}{ }^{+} \mathrm{Foxp}^{+} \mathrm{T}$ cells was tested by intranuclear staining (A, B). The level of IL-10 was detected by ELISA (C). The expression of IL-10 (D) and Foxp3 (E) mRNA was tested by RT-qPCR. Data are representative of 3 independent experiments and are expressed as the mean \pm SD. ${ }^{*} \mathrm{p}<0.05$ vs. control group; ${ }^{* *} \mathrm{p}<0.01 \mathrm{vs.}$ control group.
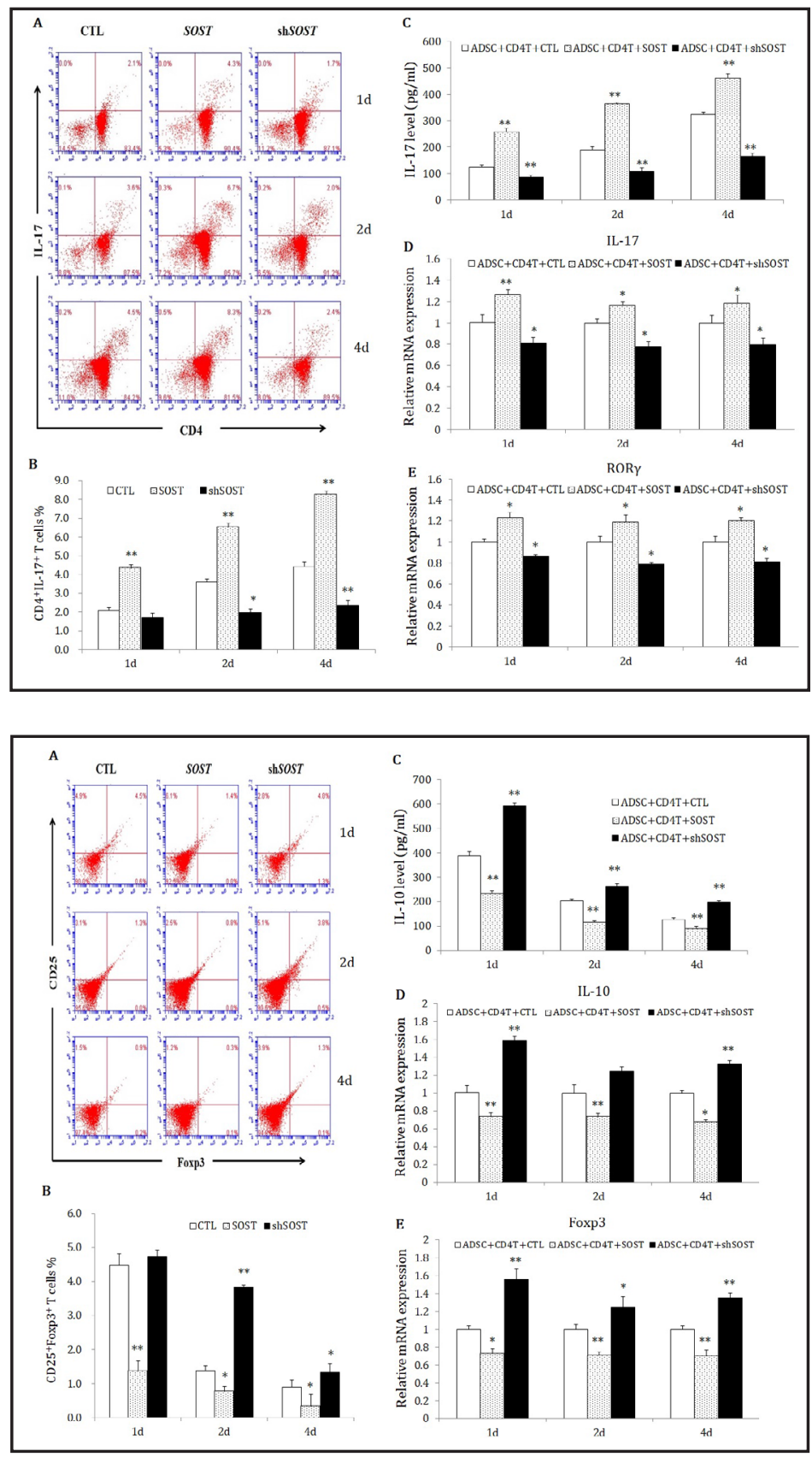
Fig. 5. Effect of SOST on osteogenesis after co-culture of ADSCs with $\mathrm{CD}^{+} \mathrm{T}$ cells. Mouse ADSCs were transfected with pcDNA3-SOST or shSOST and then co-cultured with $\mathrm{CD} 4^{+} \mathrm{T}$ cells. The expression of collagen (COL1) (A), OCN (B), and OPN (C) mRNA was analyzed by RT-qPCR. ALP activity was tested using a kit (D). Data are representative of 3 independent experiments and are expressed as the mean \pm SD. ${ }^{*} \mathrm{p}<0.05$ vs. control

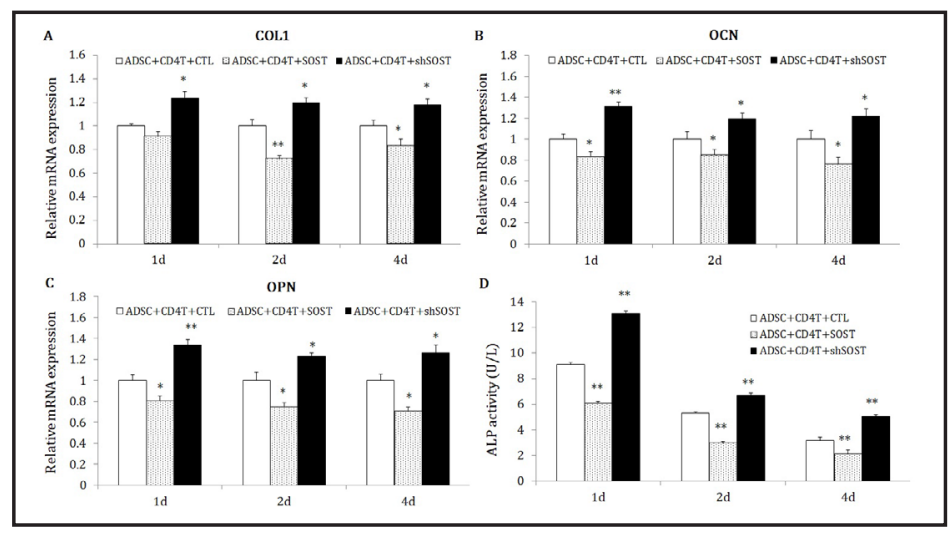
group; ${ }^{* *} \mathrm{p}<0.01$ vs. control group.

Fig. 6. Effect of SOST on adipogenesis after co-culture of ADSCs with $\mathrm{CD}^{+} \mathrm{T}$ cells. Mouse ADSCs were transfected with pcDNA3-SOST or shSOST and then co-cultured with $\mathrm{CD}^{+}{ }^{+} \mathrm{T}$ cells. The expression of LPL (A) and PPAR $\gamma$ (B) mRNA in ADSCs was analyzed by RT-qPCR. Data are representative of 3 independent experiments and are expressed as the mean \pm SD. ${ }^{*} \mathrm{p}<0.05$ vs. control group; ${ }^{* *} \mathrm{p}<0.01$ vs. control group.

\section{IL-17 promotes the SOST-induced reduction osteogenesis in ADSCs}

The above results showed that SOST promoted the expression and secretion of IL-17, which is an important cytokine for Th17 function. In this study, we further investigated the role of IL-17 on osteoblast differentiation

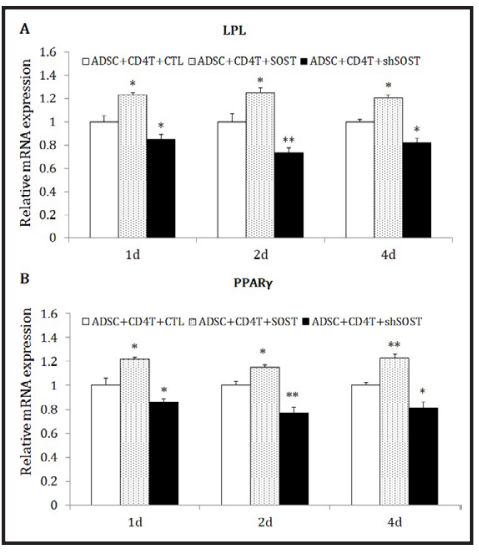
in ADSCs. ADSCs transfected with SOST or shSOST and then treated with IL-17. As shown in Fig. 7A, 7B, and 7C, similar with the effect of SOST, IL-17 treatment reduced the expression of collagen, OCN, and OPN. SOST gene transfection inhibited the IL-17-induced reduction of osteoblast differentiation. However, SOST gene knockdown with shSOST recovered the reduction of osteoblast differentiation induced by IL-17 treatment. In addition, we also found that CD4 ${ }^{+} \mathrm{T}$ cells or IL-17 treatment increased the expression of SOST gene in ADSCs (Fig. 7D).

\section{IL-17 promotes SOST-induced adipogenic differentiation in ADSCS}

Genes related to adipogenic differentiation were tested by qPCR in ADSCs after treatment with IL-17. SOST increased the expression of LPL and PPAR $\gamma$ mRNA. IL-17 treatment also increased the expression of LPL and PPAR $\gamma$ mRNA in ADSCs. SOST gene over-expression promoted IL-17-induced adipogenic differentiation. In contrast, SOST inhibition reduced IL17-induced adipogenic differentiation (Fig. 8A, B).

\section{Discussion}

MSCs from various sources constitute the basis for tissue engineering, and these cells can be differentiated into osteoblasts, adipocytes, and chondrocytes. The multipotency of ADSCs makes them a valuable and high yield source for cell-based therapeutic applications. Under specific conditions, ADSCs differentiate into an osteoblast lineage [18]. In addition, MSCs also exert broad immunomodulatory effects by their interactions with other cells. Accumulated data have demonstrated the quantitative or functional imbalance between Th17 and Treg cells in bone-related diseases, suggesting that Th17 and Treg cells are involved 
Fig. 7. Effect of IL-17 on SOSTinduced inhibition of osteogenesis. Mouse ADSCs were transfected with pcDNA3-SOST or shSOST. Then, the cells were treated with IL-17 (10 ng/mL or $100 \mathrm{ng} / \mathrm{mL}$ ). The expression of COL1 (A), OCN (B), OPN (C), and SOST (D) mRNA was analyzed by RT-qPCR. Data are representative of 3 independent experiments and are expressed as the mean \pm SD. ${ }^{*} \mathrm{p}<0.05$ vs. control group; $^{* *} \mathrm{p}<0.01$ vs. control group.

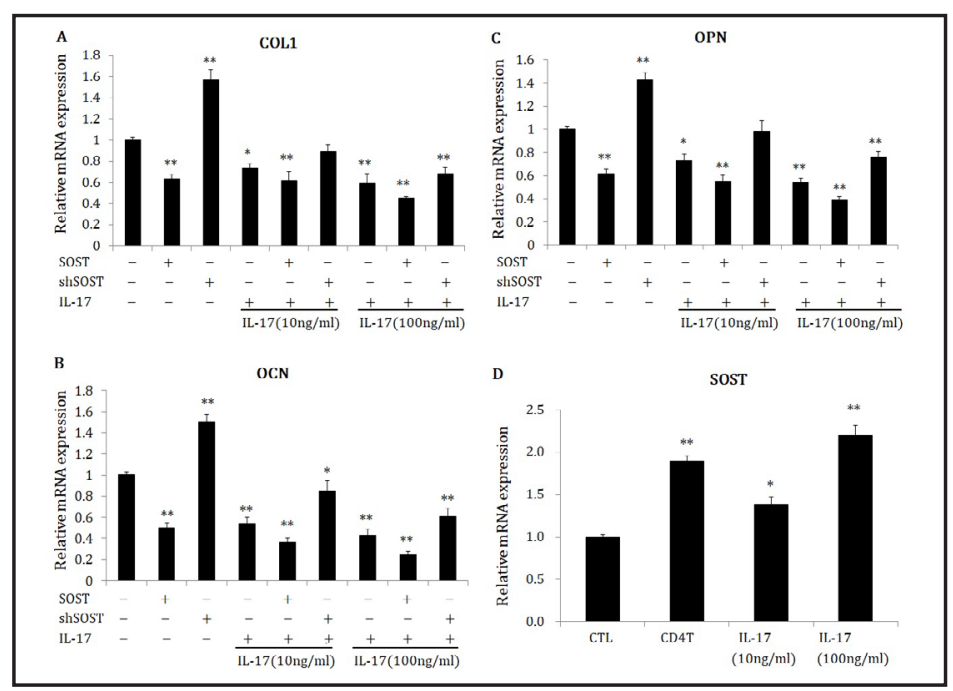

Fig. 8. Effect of IL-17 on SOST-induced adipogenesis. Mouse ADSCs were transfected with pcDNA3-SOST or shSOST. Then, the cells were treated with IL-17 (10 ng/mL or $100 \mathrm{ng} / \mathrm{mL}$ ). The expression of LPL (A) and PPAR $\gamma$ (B) mRNA was analyzed by RT-qPCR. Data are representative of 3 independent experiments and are expressed as the mean \pm SD. ${ }^{*} \mathrm{p}<0.05$ vs. control group; ${ }^{* *} \mathrm{p}<0.01$ vs. control group.

in these diseases [19]. Under inflammatory conditions, MSCs mediate the adhesion of Th17 cells via CCR6 and exert anti-inflammatory effects through the induction of a Treg phenotype in these cells [10]. SOST is an inhibitor of bone formation that induces the over-expression of the BMP inhibitor MAB21L2 in MSCs, which has been identified as a factor associated with defects in regeneration [20]. However, the role of SOST in the differentiation and function of Th17

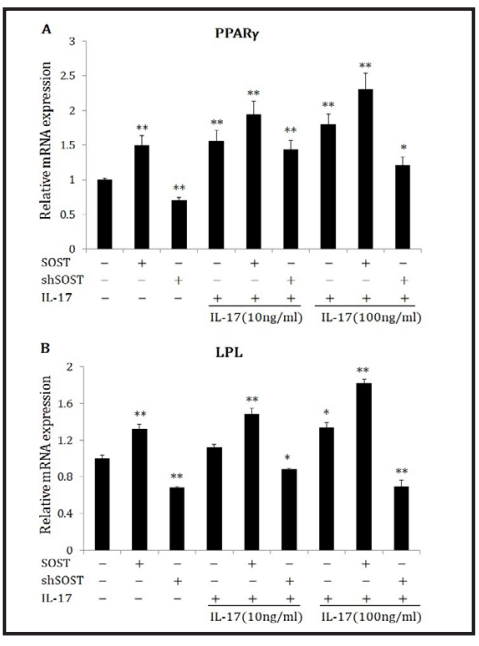
cells has not been characterized in detail, which led us to conduct the present study.

Th17 cells differentiate from naïve T cells in the presence of TGF- $\beta$ and IL- 6 in mice, or with TGF- $\beta$ plus inflammatory stimuli in humans. Th17 cells are characterized by the production of proinflammatory cytokines, including IL-17A, IL-17F, IL-21, and IL-22 [21]. IL-17, the most important effector cytokine, is involved in promoting the expression of many proinflammatory cytokines, chemokines, and mediators that contribute to inflammation. ROR $\gamma$ t is expressed specifically in Th17 cells and is required for their differentiation. ROR $\gamma \mathrm{t}$ expression is induced by the combination of IL- 6 and TGF- $\beta$ through the Stat 3 signaling pathway $[19,22]$. In the current study, SOST over-expression induced the expression and secretion of IL- 6 and TGF- $\beta$ in mouse ADSCs. These results suggested that SOST gene overexpression in ADSCs promotes the expression of cytokines related to Th17 differentiation. MSC-mediated immunomodulatory effects on T cells are known to involve cellular interactions. Inflammatory cytokines enhance CD54 expression in MSCs and result in the adherence of Th17 cells to MSCs under inflammatory conditions [10]. ADSCs can also polarize infiltrating T cells toward the pathogenic Th17 cell phenotype [23]. Serum IL-17A concentration was shown to be significantly higher in a postmenopausal osteoporosis group than in a normal postmenopausal group. These findings lead us to presume that SOST promotes the adhesion of ADSCs to T cells and induces the differentiation of Th17 cells. For this purpose, mouse CD $4^{+}$ T cells were isolated and co-cultured with ADSCs. We found that SOST gene over-expression in ADSCs promoted the differentiation of $\mathrm{CD} 4^{+} \mathrm{IL} 17^{+} \mathrm{T}$ cells, enhanced the production of IL- 
17 in PBMCs, and increased the expression of the Th17-specific transcription factor ROR $\gamma$, as well as the expression of the Th17 signature cytokine IL-17. Th17 cell differentiation and function are closely related to the development and function of Treg cells. Most evidence has indicated that the imbalance of Th17 and Treg cells plays a key role in the pathogenesis of inflammatory bone diseases [24]. Treg cell development is driven by the release of suppressor cytokines such as IL-10 and TGF- $\beta$ [25]. TGF- $\beta$ promotes Treg cell development by inducing the expression of the transcription factor Foxp3, which plays a critical role in controlling the development and functions of these cells [26]. Zhou et al. showed that Foxp3 initially inhibits Th17 differentiation by physically binding to ROR $\gamma \mathrm{t}$ [27]. In our ADSC-CD4 ${ }^{+}$ $\mathrm{T}$ cell co-culture model, SOST inhibited the differentiation of $\mathrm{CD} 4^{+} \mathrm{CD} 25^{+} \mathrm{Foxp} 3^{+}$Treg cells, decreased the production of IL-10, and reduced the expression of IL-10 and Foxp3. These data indicated that SOST gene over-expression in ADSCs promoted the differentiation of Th17 cells and reduced the differentiation of Treg cells. This may be an important factor for the development of inflammatory bone diseases.

MSCs can be differentiated either into adipocytes or osteoblasts using different factors, and the balance between these two lineages is important for bone health. Bone loss can be due to the increased bone resorption caused by osteoclasts. With aging, the composition of bone marrow shifts to favor the presence of adipocytes, osteoclast activity increases, and osteoblast function declines, resulting in osteoporosis [28]. Marrow adipocytes exert a paracrine inhibitory effect on osteoblast differentiation from mouse bone-marrow derived MSCs (BMSCs) by blocking BMP signaling [29]. ADSCs from obese subjects contribute to inflammation and reduce the insulin response in adipocytes by activating Th17 cells [30]. In the present study, we found that ADSCs promoted the differentiation of Th17 cells, which induced the production of proinflammatory factors. We examined the role of SOST in adipogenesis and osteogenesis with Th17 cell activation. After co-culture of ADSCs and CD4+ T cells, SOST reduced the expression of bone formation-related genes, such as collagen, OCN, and OPN, and decreased the activity of ALP; however, it promoted the expression of adipogenesis-related genes, such as LPL and PPAR $\gamma$. These findings indicated that SOST inhibited bone formation and promoted adipogenesis in ADSCs. IL-17 induces inflammation through the recruitment and activation of immune cells, leading to the release of proinflammatory molecules, such as IL-1 and TNF $\alpha$, which increase RANKL expression and synergize with RANKL signaling to maximize osteoclast formation [7, 31]. However, Croes et al. showed that IL-17A and IL-17F exhibit strong osteogenic effects when exposed directly to MSCs [32]. Similar to previous studies [12,33], we found that SOST reduced the expression of collagen, OCN, and OPN, which was accompanied with the increased expression of LPL and PPAR $\gamma$. However, IL-17 promoted the SOST-induced reduction of osteogenesis and increased adipogenesis in ADSCs. Inhibition of SOST gene expression blocked these effects. These results demonstrated that SOST inhibits osteogenesis by the interaction of ADSCs and Th17 cells. Interestingly, IL-17 treatment also promoted adipogenesis and reduced osteogenesis. In contrast, Ahmed and Gaffen showed that IL-17 inhibits adipogenesis by suppressing the expression of several pro-adipogenic transcription factors in 3T3-L1 cells [34]. It is possible that different cell types respond to IL-17 in different manners. In previous studies, IL-17 was shown to inhibit adipogenesis in an adipogenic cell line and MSCs. In the present study, we used ADSCs as these have been reported to have a better adipocyte differentiation capability then BMSCs [35]. In addition, Winkler et al. showed that SOST was expressed at a high level in differentiated human MSCs; however, undifferentiated human MSCs and adipocytes in adipose tissue express negligible levels of SOST [36]. SOST upregulates adipocyte differentiation in 3T3-L1 cells [33], and in our study, IL-17 treatment increased the expression of the SOST gene. According to these results, we postulate that IL17 promotes SOST gene expression in differentiated ADSC, which increases adipogenesis. However, more studies should be conducted in the future to confirm this hypothesis.

In this study, SOST gene over-expression in ADSCs induced Th17 cell differentiation and inhibited the Treg cell phenotype, which reduced osteogenesis and increased adipogenesis from ADSCs. The IL-17 proinflammatory cytokine secreted by Th17 cells aggravated 
SOST-inhibited osteogenesis. This is the first time that a role for SOST gene in Th17 cell differentiation from ADSCs has been reported, which has produced a new insight into the relationship between osteogenesis and adipogenesis in ADSCs.

\section{Acknowledgements}

This work was supported by a grant from the National Natural Science Foundation of China (No. 81570797).

Authors' roles: Li You planned the experimental design, conducted experiments, and wrote the manuscript. Lin Chen conducted experiments and flow cytometry analysis. Ling Pan analyzed data. Jinyu Chen gave technical suggestions. Yongde Peng conceived the project and wrote the manuscript.

\section{Disclosure Statement}

The authors declare to have no competing interests to disclose.

\section{References}

1 Li G, Thabane L, Papaioannou A, Ioannidis G, Levine MA, Adachi JD: An overview of osteoporosis and frailty in the elderly. BMC Musculoskelet Disord 2017;18:46.

-2 Tsuji W, Rubin JP, Marra KG: Adipose-derived stem cells: Implications in tissue regeneration. World J Stem Cells 2014;6:312-321.

-3 Liu HY, Chiou JF, Wu AT, Tsai CY, Leu JD, Ting LL, Wang MF, Chen HY, Lin CT, Williams DF, Deng WP: The effect of diminished osteogenic signals on reduced osteoporosis recovery in aged mice and the potential therapeutic use of adipose-derived stem cells. Biomaterials 2012;33:6105-6112.

4 Duffy MM, Ritter T, Ceredig R, Griffin MD: Mesenchymal stem cell effects on T-cell effector pathways. Stem Cell Res Ther 2011;2:34.

5 Mori G, D’Amelio P, Faccio R, Brunetti G: Bone-immune cell crosstalk: bone diseases. J Immunol Res 2015;2015:108451.

6 Zhao R: Immune regulation of osteoclast function in postmenopausal osteoporosis: a critical interdisciplinary perspective. Int J Med Sci 2012;9:825-832.

-7 Sato K, Suematsu A, Okamoto K, Yamaguchi A, Morishita Y, Kadono Y, Tanaka S, Kodama T, Akira S, Iwakura Y, Cua DJ, Takayanagi H: Th17 functions as an osteoclastogenic helper T cell subset that links T cell activation and bone destruction. J Exp Med 2006;203:2673-2682.

-8 Zhang J, Fu Q, Ren Z, Wang Y, Wang C, Shen T, Wang G, Wu L: Changes of serum cytokines-related Th1/Th2/ Th17 concentration in patients with postmenopausal osteoporosis. Gynecol Endocrinol 2015;31:183-190.

-9 Talaat RM, Sidek A, Mosalem A, Kholief A: Effect of bisphosphonates treatment on cytokine imbalance between TH17 and Treg in osteoporosis. Inflammopharmacology 2015;23:119-125.

10 Ghannam S, Pene J, Moquet-Torcy G, Jorgensen C, Yssel H: Mesenchymal stem cells inhibit human Th17 cell differentiation and function and induce a T regulatory cell phenotype. J Immunol 2010;185:302-312.

11 Yavropoulou MP, Xygonakis C, Lolou M, Karadimou F, Yovos JG: The sclerostin story: from human genetics to the development of novel anabolic treatment for osteoporosis. Hormones (Athens) 2014;13:323-337.

12 Li X, Ominsky MS, Niu QT, Sun N, Daugherty B, D’Agostin D, Kurahara C, Gao Y, Cao J, Gong J, Asuncion F, Barrero M, Warmington K, Dwyer D, Stolina M, Morony S, Sarosi I, Kostenuik PJ, Lacey DL, Simonet WS, Ke HZ, Paszty C: Targeted deletion of the sclerostin gene in mice results in increased bone formation and bone strength. J Bone Miner Res 2008;23:860-869.

13 van Bezooijen RL, Svensson JP, Eefting D, Visser A, van der Horst G, Karperien M, Quax PH, Vrieling H, Papapoulos SE, ten Dijke P, Lowik CW: Wnt but not BMP signaling is involved in the inhibitory action of sclerostin on BMP-stimulated bone formation. J Bone Miner Res 2007;22:19-28. 


\section{Cellular Physiology Cell Physiol Biochem 2018;48:1030-1040 \begin{tabular}{l|l} 
DOI: 10.1159/000491971 & O 2018 The Author(s). Published by S. Karger AG, Basel \\
www.karger.com/cpb
\end{tabular}}

You et al.: SOST and Th17

14 Lewiecki EM: Role of sclerostin in bone and cartilage and its potential as a therapeutic target in bone diseases. Ther Adv Musculoskelet Dis 2014;6:48-57.

15 You L, Chen L, Pan L, Gu WS, Chen JY: Zinc finger protein 467 regulates Wnt signaling by modulating the expression of sclerostin in adipose derived stem cells. Biochem Biophys Res Commun 2015;456:598-604.

16 Horowitz MC, Fretz JA: Sclerostin: A new mediator of crosstalk between the skeletal and immune systems. J Bone Miner Res 2012;27:1448-1450.

17 Zuk PA, Zhu M, Mizuno H, Huang J, Futrell JW, Katz AJ, Benhaim P, Lorenz HP, Hedrick MH: Multilineage cells from human adipose tissue: implications for cell-based therapies. Tissue Eng 2001;7:211-228.

18 Knippenberg M, Helder MN, Doulabi BZ, Semeins CM, Wuisman PI, Klein-Nulend J: Adipose tissuederived mesenchymal stem cells acquire bone cell-like responsiveness to fluid shear stress on osteogenic stimulation. Tissue Eng 2005;11:1780-1788.

19 Wang M, Tian T, Yu S, He N, Ma D: Th17 and Treg cells in bone related diseases. Clin Dev Immunol DOI:10.1155/2013/203705

20 Benisch P, Schilling T, Klein-Hitpass L, Frey SP, Seefried L, Raaijmakers N, Krug M, Regensburger M, Zeck S, Schinke T, Amling M, Ebert R, Jakob F: The transcriptional profile of mesenchymal stem cell populations in primary osteoporosis is distinct and shows overexpression of osteogenic inhibitors. PLoS One 2012;7:e45142.

21 Lee YK, Mukasa R, Hatton RD, Weaver CT: Developmental plasticity of Th17 and Treg cells. Curr Opin Immunol 2009;21:274-280.

-22 Ivanov, II, McKenzie BS, Zhou L, Tadokoro CE, Lepelley A, Lafaille JJ, Cua DJ, Littman DR: The orphan nuclear receptor RORgammat directs the differentiation program of proinflammatory $\mathrm{IL}-17^{+} \mathrm{T}$ helper cells. Cell 2006;126:1121-1133.

23 Chehimi M, Vidal H, Eljaafari A: Pathogenic Role of IL-17-Producing Immune Cells in Obesity, and Related Inflammatory Diseases. J Clin Med 2017;6:68.

24 Diller ML, Kudchadkar RR, Delman KA, Lawson DH, Ford ML: Balancing Inflammation: The Link between Th17 and Regulatory T Cells. Mediators Inflamm 2016;2016:6309219.

25 Curotto de Lafaille MA, Lafaille JJ: Natural and adaptive foxp3+ regulatory T cells: more of the same or a division of labor? Immunity 2009;30:626-635.

26 Allan SE, Passerini L, Bacchetta R, Crellin N, Dai M, Orban PC, Ziegler SF, Roncarolo MG, Levings MK: The role of 2 FOXP3 isoforms in the generation of human CD4+ Tregs. J Clin Invest 2005;115:3276-3284.

27 Zhou L, Lopes JE, Chong MM, Ivanov, II, Min R, Victora GD, Shen Y, Du J, Rubtsov YP, Rudensky AY, Ziegler SF, Littman DR: TGF-beta-induced Foxp3 inhibits T(H)17 cell differentiation by antagonizing RORgammat function. Nature 2008;453:236-240.

28 Rosen CJ, Bouxsein ML: Mechanisms of disease: is osteoporosis the obesity of bone? Nat Clin Pract Rheumatol 2006;2:35-43.

-29 Abdallah BM: Marrow adipocytes inhibit the differentiation of mesenchymal stem cells into osteoblasts via suppressing BMP-signaling. J Biomed Sci 2017;24:11.

30 Eljaafari A, Robert M, Chehimi M, Chanon S, Durand C, Vial G, Bendridi N, Madec AM, Disse E, Laville M, Rieusset J, Lefai E, Vidal H, Pirola L: Adipose Tissue-Derived Stem Cells From Obese Subjects Contribute to Inflammation and Reduced Insulin Response in Adipocytes Through Differential Regulation of the Th1/ Th17 Balance and Monocyte Activation. Diabetes 2015;64:2477-2488.

-31 Kim KW, Kim HR, Kim BM, Cho ML, Lee SH: Th17 cytokines regulate osteoclastogenesis in rheumatoid arthritis. Am J Pathol 2015;185:3011-3024.

-32 Croes M, Oner FC, van Neerven D, Sabir E, Kruyt MC, Blokhuis TJ, Dhert WJ, Alblas J: Proinflammatory T cells and IL-17 stimulate osteoblast differentiation. Bone 2016;84:262-270.

33 Ukita M, Yamaguchi T, Ohata N, Tamura M: Sclerostin Enhances Adipocyte Differentiation in 3T3-L1 Cells. I Cell Biochem 2016;117:1419-1428.

34 Ahmed M, Gaffen SL: IL-17 inhibits adipogenesis in part via C/EBPalpha, PPARgamma and Kruppel-like factors. Cytokine 2013;61:898-905.

-35 Lotfy A, Salama M, Zahran F, Jones E, Badawy A, Sobh M: Characterization of mesenchymal stem cells derived from rat bone marrow and adipose tissue: a comparative study. Int J Stem Cells 2014;7:135-142.

-36 Winkler DG, Sutherland MK, Geoghegan JC, Yu C, Hayes T, Skonier JE, Shpektor D, Jonas M, Kovacevich BR, Staehling-Hampton K, Appleby M, Brunkow ME, Latham JA: Osteocyte control of bone formation via sclerostin, a novel BMP antagonist. EMBO J 2003;22:6267-6276. 